symptom. Mean lung function values were as follows; mean (SD) percentage predicted $\mathrm{FEV}_{1} 98.1$ (15.2) and FVC 102.4 (13.9).

Fourteen workers had measured airways obstruction (as defined by an $\left.\mathrm{FEV}_{1} / \mathrm{FVC}<0.7\right)$; in this cross sectional analysis its presence did not significantly relate to current smoking status or lifetime duration of RCS exposure, although was significantly associated with an increased time worked in the current work area. Airways obstruction was also associated with the reporting of a diagnosis of (ever having) asthma and wheeze in the last 12 months.

Conclusions This cross sectional study of silica exposed brick workers has identified a cohort for longer term follow up. Future work will allow the development of dose response relationships, corrected for other relevant factors, between cumulative RCS exposure and $\mathrm{FEV}_{1}$ decline and will assist in the development of workplace interventions to reduce the health risks associated with RCS exposure in this group of workers.

\section{P62 A COMPARISON OF THE RELATIVE EFFECTS OF EXPOSURE ON FEV1 AND FVC IN OCCUPATIONAL COPD}

${ }^{1} \mathrm{JG}$ Macfarlane, ${ }^{2}$ SC Stenton. 'Northumbria Healthcare NHS Trust, Newcastle Upon Tyne, UK; ${ }^{2}$ Newcastle Hospitals NHS Foundation Trust, Newcastle Upon Tyne, UK

\subsection{6/thoraxinl-2015-207770.199}

Introduction and objectives A wide range of occupational exposures to dusts and fumes are known to cause COPD. Little is known about the underlying pathophysiology as few studies have measured gas transfer or included CT scans. It is not known whether the relative degree of small airway disease and emphysema is similar to that seen with smoking or varies by causative agents. The modifying effect on lung function of pneumonoconiosis, that is a feature of several accepted or possible causes of occupational COPD, is also uncertain.

Methods We have reviewed papers investigating exposureresponse relationships for $\mathrm{FEV}_{1}$ and $\mathrm{FVC}$ to a range of occupational dusts and fumes. We examined the ratio of the slopes of the regression equations relating exposure to $\mathrm{FEV}_{1}$ and $\mathrm{FVC}$ for the occupational exposures and for cigarette smoking.

Results We identified 15 papers dealing with exposure to coal mine dust (5 papers), silica-containing dusts (8 papers) and other dusts (2 papers). The relative effects of exposure on $\mathrm{FEV}_{1}$ and FVC are shown in Figure 1, together with the relative effects of smoking on $\mathrm{FEV}_{1}$ and FVC obtained from the same papers. It should be noted that as $\mathrm{FEV}_{1}$ is lower than $\mathrm{FVC}$, an equal reduction in $\mathrm{FEV}_{1}$ and $\mathrm{FVC}$ with exposure (ratio = 1) still leads to airflow obstruction. Cigarette smoking had an effect on $\mathrm{FEV}_{1}$ that was approximately twice the effect on FVC. Coal mine dust was associated with a similar ratio of effect though with greater variability. Studies on silica-containing dusts had a more equal effect on $\mathrm{FEV}_{1}$ and FVC. That was also the case for the two studies of non-silica containing dusts (carbon black and potash mining).

Conclusions COPD associated with exposure to silica-containing dusts appears to be associated with a more restrictive abnormality than COPD associated with cigarette smoking and coal dust, possibly because of a modifying effect of associated lung fibrosis.

\section{Ratio of the effect of exposure on $\mathrm{FEV}_{1}$ to the effect on FVC}

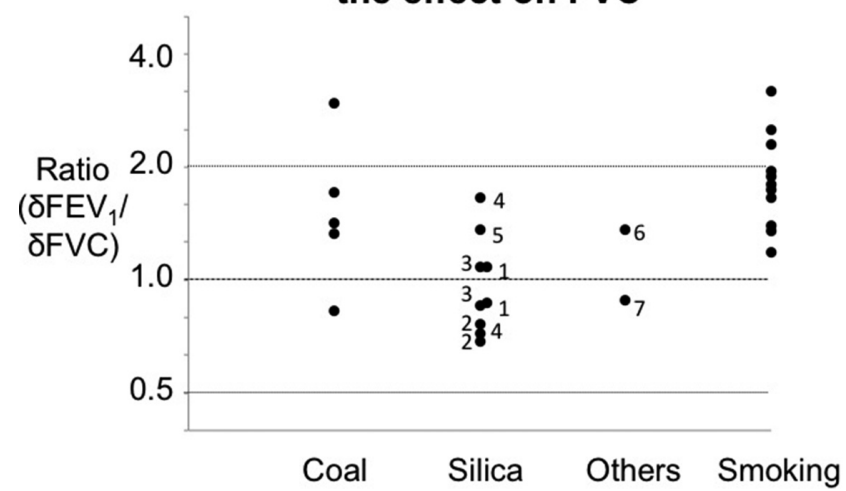

Abstract P62 Figure 1 Key to exposures: $1=$ gold mining, $2=$ foundry work, $3=$ talc processing, $4=$ mixed exposures, 5 = silicon carbide processing, $6=$ potash mining, 7 = carbon black mining

\section{P63 IS DATA QUALITY MORE IMPORTANT THAN DATA QUANTITY IN THE DIAGNOSIS OF OCCUPATIONAL ASTHMA FROM SERIAL PEAK FLOW RECORDS?}

${ }^{1}$ VC Moore, ${ }^{1}$ CBSG Burge, ${ }^{2}$ AS Robertson, ${ }^{1}$ PS Burge. 'Birmingham Heartlands Hospital, Birmingham, UK; ${ }^{2}$ University Hospitals Birmingham, Birmingham, UK

\subsection{6/thoraxjn-2015-207770.200}

Background Serial measurements of peak expiratory flow (PEF) are a recommended method for confirming a diagnosis of occupational asthma and are the only available method for low molecular weight agents available to many non-specialists. There is a tradeoff between accepting only measurements fulfilling quality standards with reduced data quantity and accepting all measurements irrespective of quality. We have investigated the effect of systematically reducing quality or quantity on the diagnostic sensitivity and specificity of these records in the diagnosis of occupational asthma using the Oasys system.

Methods Serial PEF measurements from 36 specific inhalation challenge positive occupational asthmatics and 44 non-occupational asthmatics were used. Records contained 4 weeks of $\geq 4$ PEF readings/day for $75 \%$ of days. PEFs were measured on metres without any data quality requirements. Data was corrupted in 2 ways: 1) Each PEF measurement was randomly changed to be up to $+50 \mathrm{~L} / \mathrm{min}$ or $-50 \mathrm{~L} / \mathrm{min}$ from the original value in increments of $10 \mathrm{~L} / \mathrm{min}$. Records were randomised 3 times and the sensitivity and specificity compared at each randomisation to the original using the Oasys score, area between curves $(\mathrm{ABC})$ score and timepoint analysis. 2) Independently, the number of readings per day were reduced sequentially from $\geq 7$ readings per day to 2 readings per day. The sensitivity and specificity of the Oasys score, area between curves (ABC) score and timepoint analysis were compared after each reduction.

Results Random alteration of individual readings had small effects on sensitivity and specificity at each randomisation (Table 1). When the number of readings were reduced, the sensitivity of the Oasys score and ABC score was extremely robust in all reductions down to 3 readings per day. The sensitivity of the 
timepoint analysis was more affected. With only 2 readings, sensitivity was reduced for all scores. Specificity was unaffected by data reduction (Table 1).

\begin{tabular}{|c|c|c|c|}
\hline & Oasys score & $A B C$ score & Timepoint analysis \\
\hline Pre-Randomisation & $75(84)$ & 69 (93) & $64(84)$ \\
\hline Randomisation 1 & $67(73)$ & $61(89)$ & $58(84)$ \\
\hline Randomisation 2 & $72(68)$ & $55(86)$ & $44(86)$ \\
\hline Randomisation 3 & $86(86)$ & $69(93)$ & $53(86)$ \\
\hline$\geq 7$ readings per day & $73(86)$ & $65(95)$ & $62(86)$ \\
\hline 6 readings per day & $73(80)$ & $65(100)$ & $58(81)$ \\
\hline 5 readings per day & $69(84)$ & $65(97)$ & $62(84)$ \\
\hline 4 readings per day & $73(84)$ & $69(97)$ & $54(84)$ \\
\hline 3 readings per day & $69(81)$ & $65(97)$ & $50(81)$ \\
\hline 2 readings per day & $50(86)$ & $34(97)$ & 27 (91) \\
\hline
\end{tabular}

Conclusion Specificity was not reduced by adding random errors to the peak flow measurements nor through data reduction. Sensitivity was reduced, relatively more for the timepoint analysis, but in $2 / 3$ randomisations it was preserved for the Oasys and $\mathrm{ABC}$ systems. Oasys analysis is robust despite decreasing data quality and quantity.

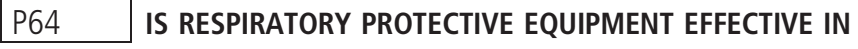 ABOLISHING THE WORK EFFECT IN PATIENTS SENSITISED TO METAL WORKING FLUID?}

${ }^{1}$ A llgaz, ${ }^{1}$ VC Moore, ${ }^{2}$ W Robertson, ${ }^{3}$ AS Robertson, ${ }^{1} P S$ Burge. ${ }^{1}$ Birmingham Heartlands Hospital, Birmingham, UK; ${ }^{2}$ University of Warwick, Coventry, UK; ${ }^{3}$ University Hospitals Bimringham, Birmingham, UK

\subsection{6/thoraxjn-2015-207770.201}

Background Removing exposure to the causative agent in workers with occupational asthma or hypersensitivity pneumonitis is the main goal. The effectiveness of Respiratory Protective Equipment (RPE) in sensitised workers with usual exposures in their workplace is not known. We aimed to assess changes in serial peak expiratory flow (PEF) in workers with sensitisation to metal working fluid before and after the introduction of RPE.

Methods Workers who had been requested to keep 2-hourly PEF measurements before and after the introduction of RPE at a particular engine manufacturing plant were searched for on the Oasys PEF database. Those who had completed a minimum of 1 week before and after the introduction of RPE and had a positive area between curves (ABC) score for occupational effect from Oasys analysis $(\geq 15 \mathrm{~L} / \mathrm{min} / \mathrm{hr})$ on either record were included. All PEF measurements were made outside the working area where the RPE could be removed. Workers who remained symptomatic after cleaning and replacement of the metal-working fluid were only allowed to continue work if they complied with the strict use of respiratory protective equipment (RPE) using powered filtration and external changing facilities.

Results 19 workers fulfilled the inclusion criteria. The mean ABC score before RPE use was $27.85 \mathrm{~L} / \mathrm{min} / \mathrm{hr}$ (SD 17.69) and after RPE use was $19.65 \mathrm{~L} / \mathrm{min} / \mathrm{hr}$ (SD 22.77) showing a mean overall decrease of $8.20 \mathrm{~L} / \mathrm{min} / \mathrm{hr}$ (SD 28.60). 9 workers (47\%) no longer showed an occupational effect on their PEF record once RPE was instated, 7 workers continued to show an occupational effect and 3 workers showed a new occupational effect which was not present before RPE use.

Conclusion Serial PEF measurements assessed with the ABC score from the Oasys system allowed quantification of the effect of RPE in sensitised workers. The RPE abolished falls in PEF associated with work exposure in $47 \%$ of workers, the remaining 10 workers had significant falls in PEF despite rigorously applied RPE. RPE should still remain a last resort in the hierarchy of control for occupational health.

\section{P65 RESPIRATORY ILL HEALTH IN THE SILICA EXPOSED STONE WORKING SECTOR}

D Fishwick, J Sumner, CM Barber, E Robinson, A Codling, L Lewis, C Young, N Warren. Centre for Workplace Health, HSL, Buxton, UK

\subsection{6/thoraxjnl-2015-207770.202}

Introduction Exposure at work to inhaled respirable crystalline silica (RCS) has previously been linked with silicosis, tuberculosis, lung cancer and COPD. Whilst the risk of developing silicosis is largely a function of cumulative lifetime RCS exposures, current workplace exposures contribute to this risk. New cases of silicosis continue to be reported in the UK. The stone working sector is one where exposures to RCS continue to place workers at risk.

Methods A cross sectional GB based workplace study of stone workers was carried out, in order to identify a subsequent longitudinal cohort. Consenting workers were asked to complete an interviewer led questionnaire, undergo lung function testing and complete a full occupational history including details of lifetime exposure to RCS. Consenting workers had a PA Chest Radiograph using a mobile facility, and levels of RCS exposure in the personal breathing zone were measured.

Results 128 workers took part; with a mean age of 40.1 years and 11 years median (range 0.5-44) years worked overall in industry. One had radiological evidence of silicosis. Respiratory symptoms were common; for example $22.7 \%$ reported cough, $33.6 \%$ wheeze in the last 12 months, $16.4 \%$ reported ever having asthma. $14.1 \%$ reported at least one work related respiratory symptom. Mean lung function values $(\mathrm{n}=127)$ were as follows; mean (SD) percentage predicted $\mathrm{FEV}_{1} 97.5$ (14.5) and FVC 103.6 (12.2).

Twenty four workers had measured airways obstruction (as defined by an $\left.\mathrm{FEV}_{1} / \mathrm{FVC}<0.7\right)$; in this cross sectional analysis its presence did not significantly relate to current smoking status or duration of RCS exposure, although was associated with the reporting of a diagnosis of (ever having) asthma or chest tightness and difficulty in breathing.

Conclusions This cross sectional study of stone workers has identified a cohort for longer term follow up. Future work will allow the development dose response relationships (using measured current, and historic, RCS levels) corrected for other relevant factors, between cumulative RCS exposure and $\mathrm{FEV}_{1}$ decline. These approaches will assist in the development of future workplace interventions to reduce the health risks associated with RCS exposure in stone workers. 